\title{
Connectivity-driven Attachment in Mobile Cellular Ad Hoc Networks
}

\author{
Julien Boite and Jérémie Leguay \\ Thales Communications \& Security, Gennevilliers, France
}

\begin{abstract}
Cellular wireless technologies (e.g. LTE) can be used to build cellular ad hoc networks. In this new class of ad hoc networks, nodes are equipped with two radio interfaces: one being a terminal, the other one being an access point. In this context, attachment decisions based on traditional criteria (e.g. signal quality) may lead to network partitions or suboptimal path lengths, thus making access point selection critical to ensure efficient network connectivity. This paper proposes a distributed greedy attachment strategy to reach near optimal network connectivity.
\end{abstract}

Keywords: Cellular Technologies, Ad Hoc Networks, Attachment Strategy, Optimal Network Connectivity

\section{Introduction}

Traditionally, ad hoc networks have been conceived and deployed with wireless equipments that use a dedicated ad hoc radio mode. However, it has been shown to be promising $[1,2,3]$ to consider cellular ad hoc networks, where cellular technologies such as LTE or WiFi (infrastructure) are used in a specific way to build ad hoc networks. In cellular ad hoc networks, nodes are composed of two radio interfaces as presented in Fig. 1(a): one acting as a terminal, the other one acting as an access point. Using either WiFi or LTE, this combination allows each node (i) to attach to only one of its neighbors using the terminal interface, and (ii) to receive many connections from neighboring nodes thanks to the access point radio interface. The network links are thus built from the attachments that nodes initiate with one another using their terminal interface. A routing protocol makes multi-hop communications possible between connected nodes. Due to nodes mobility, attachments may change over time, leading to a dynamic network topology. Therefore, the attachment decisions have to be made with caution since they directly impact the global network connectivity.

Off-the-shelf terminal interfaces generally select an access point based on pre-configured preferences and signal strength maximization. However, they do not consider connectivity at the network scale. This may

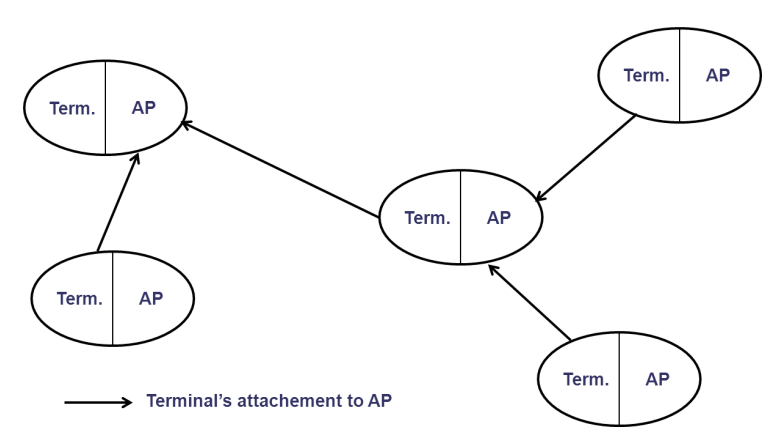

(a) Structure of a cellular ad hoc network.

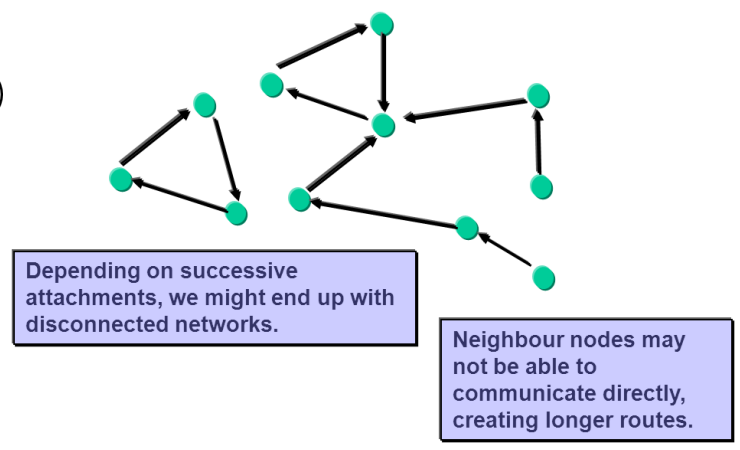

(b) Connectivity issues with signal-based attachment strategies.

Fig. 1: In cellular ad hoc network, network connectivity problems may arise with traditional attachment strategies. 
lead to problematic connectivity conditions as shown in Fig. 1(b). The network may (i) be disconnected while the radio range would allow full connectivity, or may (ii) suffer from long routes between nodes that could be closer in the network space.

We propose a distributed attachment mechanism that significantly improves network connectivity. Each node periodically runs a greedy algorithm that makes smaller network partitions attach to larger ones. By simulation, we compare our greedy approach with the optimal solution (pure ad hoc) and the state of the art solution (signal strength based attachment strategy). Our mechanism outperforms traditional attachment strategies and offers close to optimal network connectivity.

\section{A Greedy Attachment Strategy}

The main objective of the mechanism we propose is to maximize the number of nodes that can communicate. For this, we adopt a greedy attachment strategy that dynamically connects nodes to their best connected neighbors. The decisions are made locally by each node, using two sources of information: (i) the network topology that nodes maintain, retrieved e.g. from OLSR and limited to the network part they belong to, and (ii) the size (number of nodes) of neighboring nodes' connected component ${ }^{\dagger}$, exchanged between nodes through existing fields (SSID for WiFi, PLMN for LTE) during the neighbor discovery process. As a secondary purpose, the algorithm tries (i) to minimize the number of flows that may be impaired by reattachment decisions, and (ii) to improve the connectivity of already connected areas by minimizing the average path length. Overall, the attachment decisions trade off between improving connectivity (increasing the number of connected pairs of nodes, reducing the average path length) and reducing flow impairments.

The attachment procedure is greedy as it permanently tries to increase the size of nodes' connected component. Algorithm 1 details this procedure that applies in several cases: (i) periodically when nodes are not attached to any neighbor, (ii) immediately after a node loses its attachment, and (iii) periodically when nodes are already attached with the aim to further increase connectivity, reduce path length, or update the topology according to the mobility of nodes. The algorithm evaluates the interest of attaching to a neighbor with regards to the global network connectivity to make an attachment decision. It considers the following factors:

1. Opportunity to increase the size (number of nodes) of the node's connected component. The attachment strategy first looks for a neighbor outside the node's connected component that would allow to increase the number of nodes that can be reached. Since the decision to modify an attachment can disrupt or impact ongoing flows (packet losses, increased path length and delays), the attachment strategy estimates this impact and finally decides whether or not to change the attachment.

2. Opportunity to reduce paths length inside the node's connected component. When the size of the connected component cannot be increased, the attachment strategy looks for a neighbor that would substantially reduce paths length in the connected component to optimize network connectivity. The function optimizePathLengthInsideCc() in Algorithm 1 implements this process.

Two thresholds are used to control reattachments: (i) thresholdFlowDisruptions prevents handovers that would impair too many flows, and (ii) thresholdPathLengthGain (inside function optimizePathLengthInsideC ()$)$ prevents handovers that would not generate a sufficient gain on the average path length. The combination of these two thresholds allows to trade off between the improvement of connectivity and the negative impact that frequent changes of attachments may have. Due to space limitation, we do not discuss their impact, but results we obtained from extensive simulations show that using small values for those thresholds eliminates low value handovers while keeping near-optimal network connectivity.

\section{Performance Evaluation}

We have evaluated our distributed attachment mechanism in a simulator that we have developed. It generates reproducible mobility scenarios using the classical random waypoint model and simulates the progressive

\footnotetext{
$\dagger$ We call connected component of a node the network area composed of reachable nodes
} 


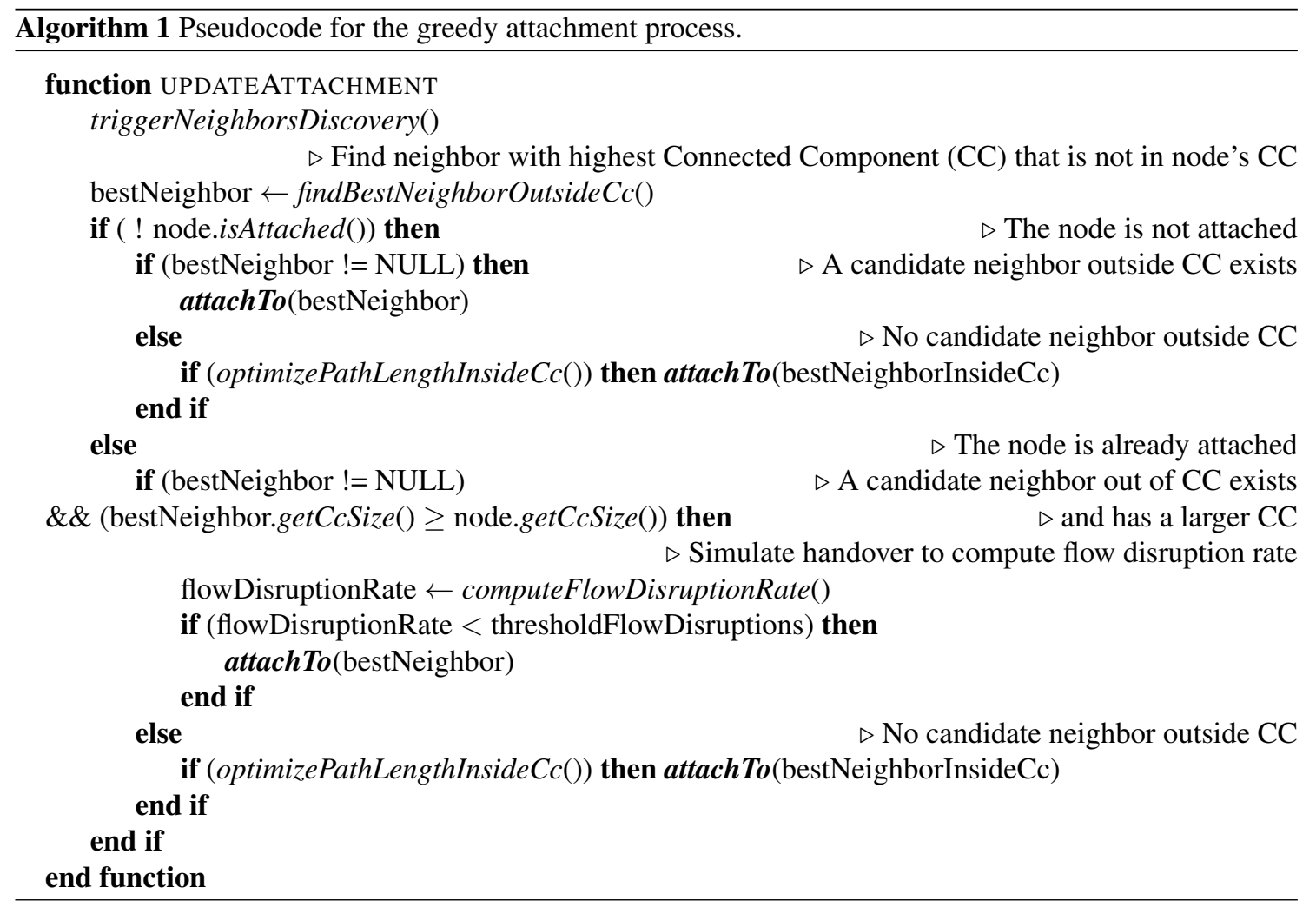

attachments between nodes. We performed several simulations to analyze the network connectivity obtained using the following attachment strategies:

- Signal: a strategy that selects the neighbor with the highest signal quality

- Optimal: a strategy that provides the best ad hoc connectivity. This strategy simulates the traditional ad hoc interface where nodes can exchange data as soon as they are in radio range (i.e. without the constraint of the cellular mode, where one node can only attach to one of its neighbors)

- Greedy: the greedy strategy that we propose.

To evaluate the performance of these different strategies, we have considered a scenario with 25 nodes moving in a $600 \mathrm{~m} \times 600 \mathrm{~m}$ area during 1 minute. Each node has a radio range of $120 \mathrm{~m}$ and moves at random speeds in the interval [0:30] $\mathrm{m} / \mathrm{s}$. We finally observed the following measures: (i) the percentage of connected pairs of nodes (i.e. for which an IP path exists), and (ii) the average path length between connected nodes, in number of radio hops. Fig. 2 presents several simulation results. We can observe that:

- the percentages of connected pairs of nodes for Greedy and Optimal are very close. We analyzed that the average percentage of connected pairs of nodes (over the whole simulation) for Greedy, Signal and Optimal is respectively $70.4 \%, 59.8 \%$ and $73.2 \%$. The greedy strategy is thus very close to optimal while Signal leads to less than $60 \%$ of pairs of nodes connected in average. This confirms the network connectivity problem that occurs with traditional attachment strategies, and demonstrates the efficiency of our greedy strategy.

- the average path length for Greedy is higher than for Optimal, but most of the time lower than for Signal. We analyzed that the average path length is 1.16 hop longer for Greedy than Optimal for which it is 2.33 hops in average. This path elongation is due to the connectivity constraint (one node can attach to only one other node) that does not exist in pure ad hoc mode. Greedy does not reach Optimal due to this constraint, but it still performs better than Signal regarding average path length. 


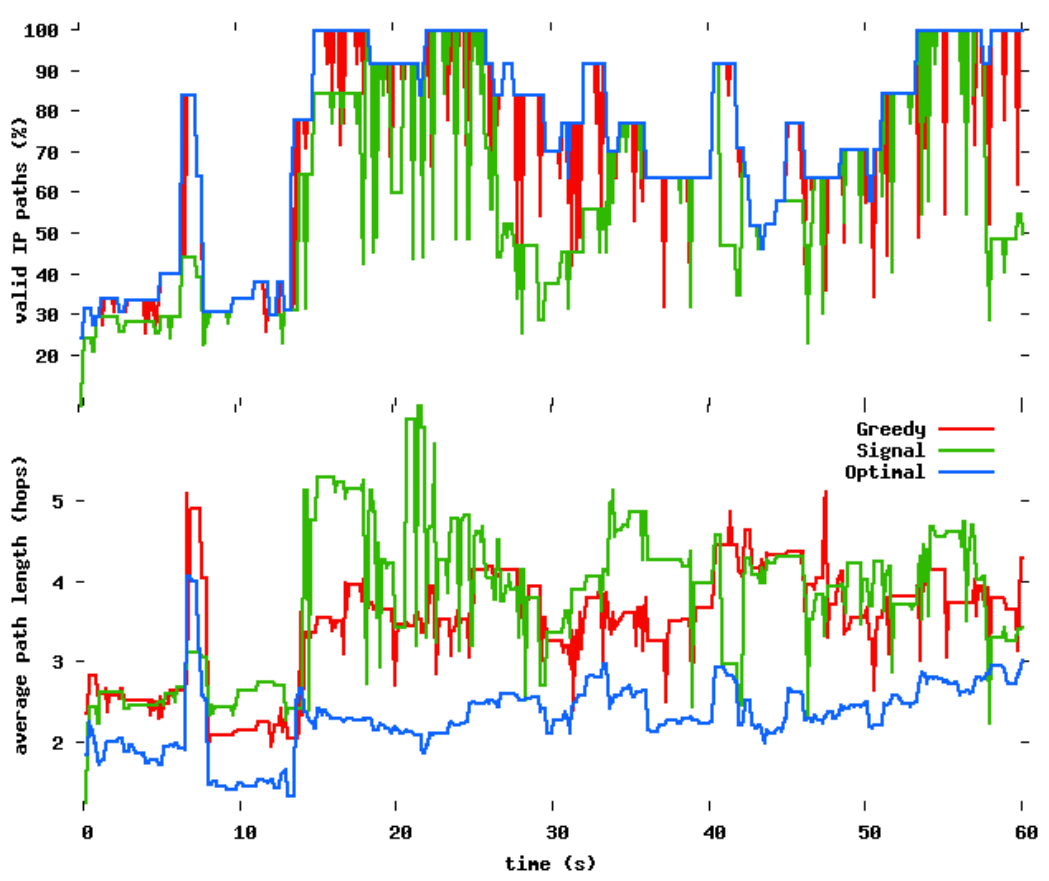

Fig. 2: Percentage of connected pairs of nodes (top) and average path length (bottom) over time.

These results show that our greedy attachment strategy outperforms traditional access point selection schemes in terms of network connectivity between nodes. It provides connectivity performances very near to those of a traditional ad hoc network. The single attachment constraint only results in slightly longer average path length compared to the optimal.

\section{Conclusions and Perspectives}

This paper has presented a greedy attachment strategy in order to improve the global network connectivity in mobile ad hoc networks built with cellular wireless technologies. Our strategy is driven by the objective of maximizing the number of nodes that can communicate so that the network connectivity is the best possible. It can be implemented on top of WiFi or LTE cellular technologies and takes benefit from existing control channels to exchange connectivity information (size of the connected component) between nodes with no overhead. Through simulations, we show that our strategy leads to a network connectivity almost as good as in pure ad hoc networks, while the average path length is a bit longer. The evaluation we made has shown that this strategy gives results close to the optimal and outperforms the traditional signal strength based strategy. The thresholds that can be configured to control reattachments allow to trade off between best connectivity and the impact of those reattachments.

The attachment strategy presented here may be included in a large multi-criteria algorithm, taking for instance into account signal strength, the social proximity of nodes as it influences traffic patterns, or the number of terminals already attached to a node. We plan to study such complex decisions in future work.

\section{References}

[1] H. Wirtz, T. Heer, R. Backhaus, and K. Wehrle, "Establishing mobile ad-hoc networks in 802.11 infrastructure mode," in ACM Chants, 2011.

[2] P. Bereski and A. Soulie, "Flexible radio network," http://patent.ipexl.com/EP/1936872-a1.html, 2008.

[3] M. H. Sarshar, P. K. Hoong, and I. Abdurrazaq, "NodesJoints: A Framework for Tree-Based MANET in IEEE 802.11 Infrastructure Mode," in IEEE Symp. on Computers and Informatics, 2013. 